\title{
Work Engagement as a Measure of Wellness in Pediatric Hospital Medicine
}

\author{
Elise P. Lu ${ }^{\mathrm{a}}$, Kathryn Leyens ${ }^{\mathrm{b}}$, Tony Tarchichia ${ }^{\mathrm{a}}$, Sylvia Choi ${ }^{\mathrm{a}}$, \\ Sara McIntire ${ }^{\mathrm{a}}$, Andrew McCormick ${ }^{\mathrm{a}, \mathrm{c}}$
}

\begin{abstract}
Background: Literature on wellness in pediatrics is limited and there are no published data specific to pediatric hospital medicine (PHM). Existing literature on wellness focuses largely on physician burnout, but wellness also includes positive traits like work engagement. We sought to assess work engagement and burnout in pediatric hospitalists nationwide.
\end{abstract}

Methods: The study utilized a survey including demographic data, the Utrecht Work Engagement Scale (UWES-17), and the Mini-Z burnout measure. The survey recruited participants via the American Academy of Pediatrics Section on Hospital Medicine Listserv.

Results: Totally, 432 of 3,085 (14\%) respondents completed the survey with mean total UWES score of 4.36 and $36 \%$ reported burnout. As expected, higher work engagement scores correlated with decreased rates of burnout $(\mathrm{P}<0.0005)$. Interestingly, work engagement varied by gender and career stage, with lowest scores found in women in early to mid-career $(\mathrm{P}<0.05)$.

Conclusions: In this study we evaluated the wellness of pediatric hospitalists, a group that has not been previously studied, using a combination of burnout and work engagement measures which, while validated, had not been previously used to evaluate physician wellness. This study suggests that wellness interventions could be most effective if targeting women in early to mid-career. Further study is needed to determine causes of decreased work engagement and consider appropriate interventions.

Keywords: Wellness; Pediatric hospital medicine; Burnout

Manuscript submitted July 31, 2020, accepted August 7, 2020

Published online August 21, 2020

aPaul C. Gaffney Division of Pediatric Hospital Medicine, UPMC Children's Hospital of Pittsburgh, Pittsburgh, PA, USA

bMedicine/Pediatrics Residency Program, UPMC Children's Hospital of Pittsburgh, Pittsburgh, PA, USA

${ }^{\mathrm{c} C o r r e s p o n d i n g ~ A u t h o r: ~ A n d r e w ~ M c C o r m i c k, ~ D i v i s i o n ~ o f ~ P e d i a t r i c ~ H o s p i t a l ~}$ Medicine, Third Floor Faculty Pavilion, Children's Hospital of Pittsburgh, 4401 Penn Ave, Pittsburgh, PA 15224, USA. Email: mccormickaa@upmc.edu

doi: https://doi.org/10.14740/ijcp399

\section{Introduction}

Physician wellness and its promotion have gained increased attention in recent years. According to Brady et al (2018) [1], "wellness is a multifaceted construct that includes mental, physical, social and spiritual quality of life in both physicians' work and personal lives." It represents a balance between positive attributes like vigor and job satisfaction, and negative factors such as burnout $[1,2]$. While physician wellness is important for its own sake, evidence also shows that well physicians provide better care. Indicators of physician wellness are associated with increased patient satisfaction, improved communication, and even increased adherence to medical recommendations [1].

Most studies of physician wellness have focused on measuring burnout, a "state of mental and physical exhaustion related to work or care-giving activities [3]." Burnout has been associated with increased medical errors, inadequate communication, and inappropriate discharges [3-5]. Factors associated with burnout include higher workloads, decreased social support from colleagues, and less autonomy [6]. Burnout is highest in early and mid-career physicians and in family medicine, internal medicine, and surgery $[1,2,4,6]$.

Rates of burnout in pediatrics are comparable to or slightly lower than other specialties. In recent AAP surveys, 22-30\% of pediatricians reported currently experiencing burnout $[6$, 7]. However, burnout varies by subspecialty within pediatrics, with much higher rates in neonatology, pediatric critical care medicine, and hematology oncology when compared with general pediatrics $[4,5,7]$. Pediatric hospitalists have been grouped with outpatient general pediatrics, and no data exist on burnout specifically of pediatric hospitalists.

There are limited data on positive aspects of wellness for pediatricians as well. One such positive aspect is work engagement, defined as "a positive, fulfilling work-related state of mind that is characterized by vigor, dedication, and absorption" [8]. The Utrecht Work Engagement Scale (UWES) is a validated tool that is used to measure work engagement [9]. Measuring work engagement would allow for better exploration of physician wellness and more tailored interventions to improve both physician and patient outcomes.

In this study, we have measured the wellness of pediatric hospitalists across the country by assessing both work engagement and burnout. This is the first such evaluation of wellness specific to the rapidly growing subspecialty of pediatric hos- 
pital medicine (PHM). Using this data, we have also sought to identify populations within this subspecialty that may be targets for future interventions to improve wellness.

\section{Materials and Methods}

The institutional review board (IRB) approval was obtained through the University of Pittsburgh (18120152). This study was conducted in compliance with the ethical standards of the responsible institution on human subjects as well as with the Helsinki Declaration. Participants were recruited via the American Academy of Pediatrics (AAP) Section on Hospital Medicine (SOHM) Listserv, an email list of 3,085 pediatric hospitalists. An email including a description of the study's purposes was sent with an anonymous link to the survey on the University of Pittsburgh Qualtrics server. Participant's informed consent was obtained by activation of the link. To encourage recruitment, three participants were randomly selected to each obtain a $\$ 25$ gift card. The survey included demographic variables such as gender, age, years of practice, practice type and academic rank, which were given as categorical variables in lettered choices. We utilized the UWES-17 to assess work engagement. Work engagement scores were calculated as described in prior studies, given as a total score and broken down into the individual facets of vigor, dedication, and absorption $[8,9]$. We assessed burnout with a validated, non-proprietary single-question measure, the Mini- $Z$ questionnaire [10]. This question has been validated against the Maslach Burnout Inventory (MBI). Answering 1 or 2 was scored as no burnout, answering 3, 4, or 5 was scored as burnout. The full survey has been included as an addendum with identification of the UWES-17 and Mini-Z for reference (Supplementary Material 1, www.theijcp.org).

Descriptive statistics were summarized as frequencies (percentages, \%) for categorical data or as mean (standard deviation) or median (interquartile range) for normally distributed or non-normally distributed continuous data, respectively. One-way analysis of variance (ANOVA) was used to examine associations between personal (e.g., race) or work characteristics (e.g., academic rank) and the outcome of interest (work engagement). If the overall one-way ANOVA was statistically significant, post hoc pairwise comparisons were performed. Pearson's Chi-square or Fisher's exact test, as appropriate, was used to examine associations between categorical variables and burnout. For ANOVA and Pearson's Chi-square (or Fisher's exact) tests, adjustment for multiple hypotheses testing was performed using the Bonferroni correction method. To examine whether the association between academic rank and work engagement varies by gender (i.e., effect modification), a general linear model was used and included main effects for academic rank and gender, and the interaction effect (i.e., academic rank $\mathrm{X}$ gender). The data were analyzed using SAS (version 9.3; SAS Institute, Cary, NC).

\section{Results}

Four hundred thirty-two respondents completed a question-
Table 1. Demographic and Career Data for Respondents

\begin{tabular}{|c|c|c|}
\hline & Number & Percentage \\
\hline \multicolumn{3}{|l|}{ Gender } \\
\hline Female & 341 & $79 \%$ \\
\hline Male & 91 & $21 \%$ \\
\hline \multicolumn{3}{|l|}{ Age (year) } \\
\hline $21-30$ & 23 & $5 \%$ \\
\hline $31-45$ & 315 & $73 \%$ \\
\hline $46-55$ & 58 & $13 \%$ \\
\hline $56-65$ & 27 & $6 \%$ \\
\hline$>65$ & 9 & $2 \%$ \\
\hline \multicolumn{3}{|l|}{ Race } \\
\hline White & 324 & $75 \%$ \\
\hline Black & 15 & $3 \%$ \\
\hline Asian/Pacific Islander & 68 & $16 \%$ \\
\hline Native American & 1 & $0 \%$ \\
\hline Did not answer & 24 & $6 \%$ \\
\hline \multicolumn{3}{|l|}{ Work environment } \\
\hline Community & 92 & $21 \%$ \\
\hline Academic & 251 & $58 \%$ \\
\hline Combined & 89 & $21 \%$ \\
\hline \multicolumn{3}{|l|}{ Years since residency } \\
\hline $0-5$ & 148 & $34 \%$ \\
\hline $5-10$ & 107 & $25 \%$ \\
\hline $10-15$ & 86 & $20 \%$ \\
\hline $15-20$ & 43 & $10 \%$ \\
\hline$>20$ & 48 & $11 \%$ \\
\hline \multicolumn{3}{|l|}{ Academic rank } \\
\hline Instructor & 45 & $10 \%$ \\
\hline Assistant professor & 220 & $51 \%$ \\
\hline Associated professor & 58 & $13 \%$ \\
\hline Full professor & 20 & $5 \%$ \\
\hline Not applicable & 89 & $21 \%$ \\
\hline
\end{tabular}

naire, a response rate of $14 \%$ (432 out of 3,085$)$. Of these respondents, the majority were white, female, aged $31-45$, worked in an academic environment, and were assistant professors. Respondent characteristics are detailed in Table 1.

The mean total score on the UWES was 4.36, significantly higher than the physician normative score of 2.10 published in the validation studies for UWES-17. SOHM members also scored higher in each individual facet of the UWES. However, the normative scores come with a significant caveat. In the initial validation studies, physicians were recruited as part of a "career monitor" program given to physicians who had experienced a career problem. Therefore, this normative score is likely much lower than the actual average for 


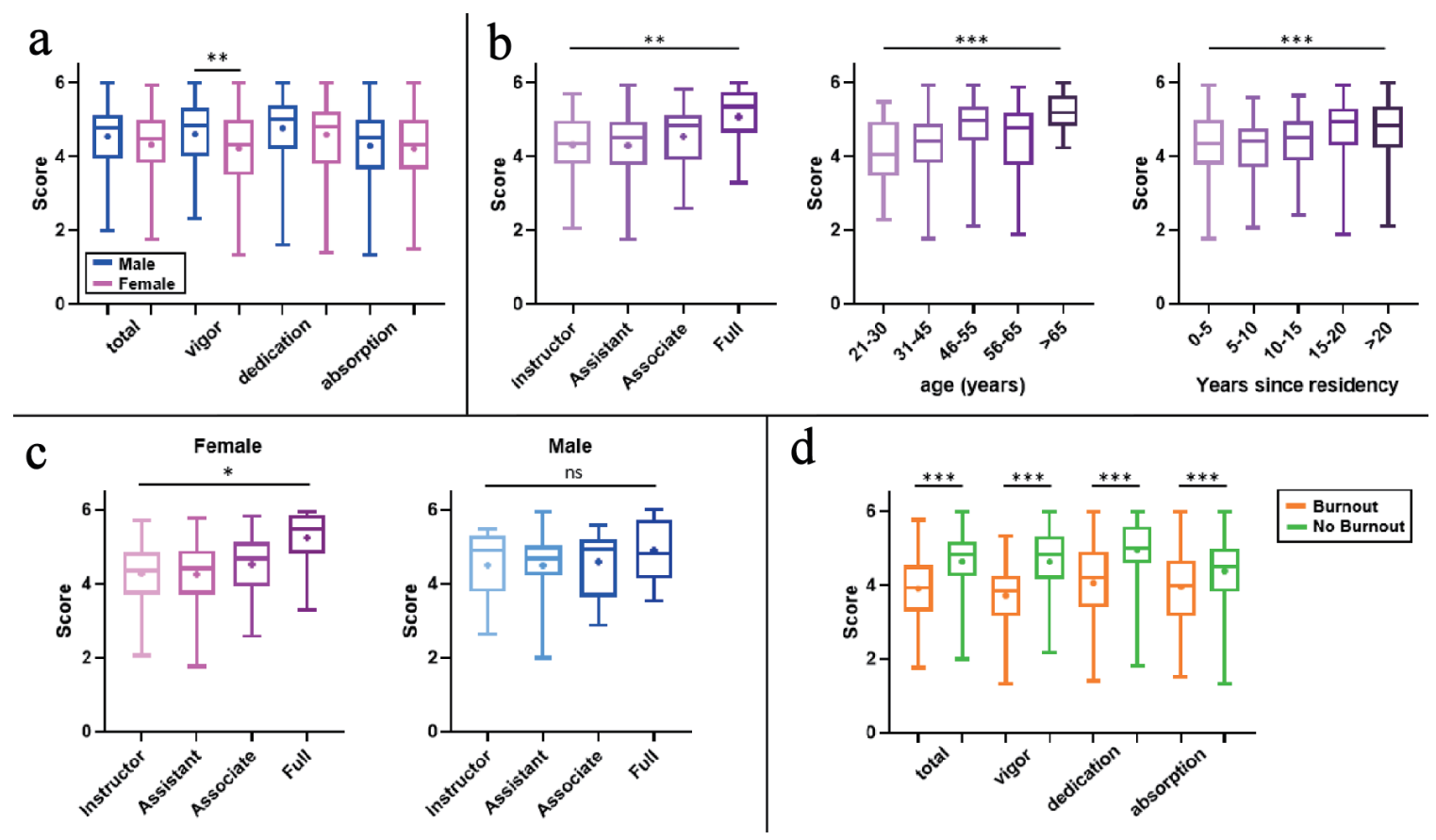

Figure 1. Work engagement varies with gender and career stage and is inversely related to burnout. (a) Men reported higher average work engagement scores than women, but this was not statistically significant when corrected for multiple comparisons. Scores for vigor showed a statistically significant difference between genders. (b) Total UWES scores increase with increasing professional rank, age, and years since residency. This progression is seen in all facets of the score. (c) The trend of increasing work engagement with career progression holds for women but is not statistically significant for men. Data are shown for rank and total UWES, but correlation holds for age and years since residency and all facets of work engagement score, as well. (d) Work engagement is the antipode of burnout. Participants who endorsed burnout had significantly lower work engagement scores in all facets compared with participants who did not endorse burnout. Statistical analysis includes Student's $t$-test and ANOVA with Bonferroni correction for multiple comparisons. For analysis of academic rank, participants who selected "not applicable" were not included in analysis. The data are plotted as a box and whiskers plot: dot indicates mean, line indicates median, box encompasses 25 th to 75 th percentiles, and whiskers show minimum and maximum values. ${ }^{*} P<0.05,{ }^{* *} P<0.005,{ }^{* * *} P<0.0005$. UWES: Utrecht Work Engagement Scale; ANOVA: analysis of variance.

all physicians.

The effects of multiple demographic and career variables on work engagement scores were assessed. Scores did not vary significantly with work environment. Total and vigor scores were higher in those who identified as white relative to those who identified as Asian, but this is difficult to interpret given the number of respondents $(24,5.5 \%)$ who declined to identify their race. Interestingly, men had significantly higher vigor scores than women (Fig. 1a). Total scores were also higher, but this was not statistically significant after correction for multiple comparisons.

The most significant variation in work engagement scores was seen with markers of career stage, including age, years since residency, and academic rank (Fig. 1b). Work engagement increased with increasing age and career stage. Interestingly, when data were broken down by multiple variables, it was found that the trend to greater work engagement with career progression held for women, but not for men. Women have increasing work engagement scores (total and all facets) with increasing age, academic rank, and years since residency, while there is no such statistically significant trend in men (Fig. 1c). Modeling interactions between rank and gender, however, was not statistically significant.
Burnout was scored as present (answer 3 or higher on single item survey) or absent (answer 2 or lower on single item survey). Thirty-six percent of respondents endorsed symptoms of burnout. Women were more likely to report burnout than men (39\% vs. $26 \%$ ), but this was not statistically significant after correction for multiple comparisons. There was also a trend to higher burnout in mid-career physicians and at younger ages, but these trends were also not statistically significant.

In combining the two components of our survey, it was found that work engagement was negatively correlated with burnout. Individuals with burnout had significantly lower work engagement scores across all facets (Fig. 1d).

\section{Discussion}

There has been a movement to both understand and improve physician wellness. This study demonstrates the first use of the UWES to evaluate wellness in pediatricians, and the first study to assess the wellness of pediatric hospitalists. Prior studies have measured wellness by looking at burnout alone; however, wellness is interplay between both positive and negative com- 
ponents, and the negative components alone may not create an accurate representation in the physician population. We combined the Mini-Z burnout measure with the UWES to create a more sensitive tool to investigate wellness of physicians. Work engagement and burnout are inversely related, as was demonstrated in our study. Survey results from SOHM members demonstrated that there were comparable levels of burnout using our survey tool to prior published data of pediatricians. This helps to provide validation of our survey tool and study methods for future use. Rates of burnout in PHM (36\%) are slightly higher than published reports of general pediatricians $(29 \%)$, but lower than other subspecialties such as critical care medicine $(71 \%)$ [5].

In this study, we determined correlations between work engagement and demographic factors. There was a positive correlation of work engagement with age, academic rank, and years since residency, indicating that engagement increases with career progression. Interestingly, this correlation only held true for women, not men. Furthermore, when comparing women to men on work engagement there was not significant difference except for in the setting of vigor. Vigor represents to aspect of work engagement associated with energy to remain connected to work.

There are weaknesses to this study that must be taken into account when interpreting the data. First, the gender difference may be due to reduced participation of men in this study and a larger sample size of men would be needed to confirm this initial finding. Second, while the overall sample size was sufficient the response rate (14\%) may reflect self-selection bias and reflect a specific subpopulation rather generalizable data for the field of PHM. Even with these weaknesses, the data highlight an important conversation around addressing physician wellness among PHM physicians specifically among woman.

Overall, a conclusion is that early to mid-career women have the lowest levels of work engagement, and inversely, the highest levels of burnout. Interesting work engagement differences seem most centered around the domain of vigor. Our study cannot extrapolate why this population is particularly at risk. However, prior studies have shown that male physicians with children have higher work engagement and less workfamily conflict than female physicians with children [11]. Physician wellness has also been positively associated with work-life balance. In a recent AAP study, female pediatricians were more likely than males to have primary responsibility for household responsibilities, including childcare [12]. Early to mid-career female pediatricians also earned less than their male counterparts [13]. Increased work-home demands with decreased compensation could explain the variation in work engagement by gender and career stage. Future work should focus on identifying causes of low work engagement and high burnout in early and mid-career women and targeting interventions for this group.

\section{Supplementary Material}

Suppl 1. National Pediatric Hospital Medicine Wellness Survey.

\section{Acknowledgments}

Institutional funding provided via UPMC Thrive physician wellness grant. No external funding was used.

\section{Financial Disclosure}

Elise P. Lu, Kathryn Leyens, Tony Tarchichi, Sylvia Choi, Sara McIntire, and Andrew McCormick have indicated they have no financial relationships relevant to the article to disclose.

\section{Conflict of Interest}

Elise P. Lu, Kathryn Leyens, Tony Tarchichi, Sylvia Choi, Sara McIntire, and Andrew McCormick have indicated they have no potential conflicts of interest to disclose.

\section{Informed Consent}

The informed consent was obtained.

\section{Author Contributions}

Andrew McCormick and Tony Tarchichi designed the initial survey instrument and received the survey responses. Elise P. Lu and Kathryn Leyens performed all data analysis and Elise P. Lu wrote the manuscript. Sylvia Choi and Sara McIntire assisted in survey design and edited the manuscript.

\section{Data Availability}

The data supporting the findings of this study are available from the corresponding author upon reasonable request.

\section{References}

1. Brady KJS, Trockel MT, Khan CT, Raj KS, Murphy ML, Bohman B, Frank E, et al. What do we mean by physician wellness? A systematic review of its definition and measurement. Acad Psychiatry. 2018;42(1):94-108.

2. Blackwelder R, Watson KH, Freedy JR. Physician wellness across the professional spectrum. Prim Care. 2016;43(2):355-361.

3. Baer TE, Feraco AM, Tuysuzoglu Sagalowsky S, Williams D, Litman HJ, Vinci RJ. Pediatric resident burnout and attitudes toward patients. Pediatrics. 2017;139(3).

4. Shanafelt TD, Boone S, Tan L, Dyrbye LN, Sotile W, Satele D, West CP, et al. Burnout and satisfaction with work-life balance among US physicians relative to the general US population. Arch Intern Med. 2012;172(18):1377-1385.

5. Garcia TT, Garcia PC, Molon ME, Piva JP, Tasker RC, 
Branco RG, Ferreira PE. Prevalence of burnout in pediatric intensivists: an observational comparison with general pediatricians. Pediatr Crit Care Med. 2014;15(8):e347353.

6. Starmer AJ, Frintner MP, Freed GL. Work-life balance, burnout, and satisfaction of early career pediatricians. Pediatrics. 2016;137(4).

7. McClafferty H, Brown OW, Section on Integrative Medicine, Committee on Practice, Ambulatory Medicine, Section on Integrative Medicine. Physician health and wellness. Pediatrics. 2014;134(4):830-835.

8. Schaufeli WB, Bakker AB, Salanova M. The measurement of work engagement with a short questionnaire: a cross-national study. Educ Psychol Meas. 2006;66(4):701-716.

9. Schaufeli WB, Bakker AB. Utrecht Work Engagement
Scare: Preliminary manual. 2004.

10. Dolan ED, Mohr D, Lempa M, Joos S, Fihn SD, Nelson $\mathrm{KM}$, Helfrich CD. Using a single item to measure burnout in primary care staff: a psychometric evaluation. J Gen Intern Med. 2015;30(5):582-587.

11. Mache S, Bernburg M, Groneberg DA, Klapp BF, Danzer G. Work family conflict in its relations to perceived working situation and work engagement. Work. 2016;53(4):859-869.

12. Starmer AJ, Frintner MP, Matos K, Somberg C, Freed G, Byrne BJ. Gender discrepancies related to pediatrician work-life balance and household responsibilities. Pediatrics. 2019;144(4).

13. Frintner MP, Sisk B, Byrne BJ, Freed GL, Starmer AJ, Olson LM. Gender differences in earnings of early- and midcareer pediatricians. Pediatrics. 2019;144(4). 